\title{
Perceptually Based Appearance Modification for Compliant Appearance Editing
}

\author{
Alvin J. Law ${ }^{1}$, Daniel G. Aliaga ${ }^{1}$, Behzad Sajadi², Aditi Majumder ${ }^{2}$ and Zygmunt Pizlo ${ }^{3}$ \\ ${ }^{1}$ Department of Computer Science, Purdue University, IN, USA \\ $\{$ ajlaw, aliaga\}@cs.purdue.edu \\ ${ }^{2}$ Department of Computer Science, University of California, Irvine, CA, USA \\ \{bsajadi, majumder\}@ics.uci.edu \\ ${ }^{3}$ Department of Psychological Sciences, Purdue University, IN, USA \\ pizlo@psych.purdue.edu
}

\begin{abstract}
Projection-based appearances are used in a variety of computer graphics applications to impart different appearances onto physical surfaces using digitally controlled projector light. To achieve a compliant appearance, all points on the physical surface must be altered to the colours of the desired target appearance; otherwise, an incompliant appearance results in a misleading visualization. Previous systems typically assume to operate with compliant appearances or restrict themselves to the simpler case of white surfaces. To achieve compliancy, one may change the physical surface's albedo, increase the amount of projector light radiance available or modify the target appearance's colours. This paper presents an approach to modify a target appearance to achieve compliant appearance editing without altering the physical surface or the projector setup. Our system minimally alters the target appearance's colours while maintaining cues important for perceptual similarity (e.g. colour constancy). First, we discuss how to measure colour compliancy. Next, we describe our approach to partition the physical surface into patches based on the surface's colours and the target appearance's colours. Finally, we describe our appearance optimization process, which computes a compliant appearance that is as perceptually similar as possible to the target appearance's colours. We perform several real-world projection-based appearances and compare our results to naïve approaches, which either ignore compliancy or simply reduce the appearance's overall brightness.
\end{abstract}

Keywords: projector-camera system, augmented reality, appearance editing, radiometric calibration

ACM CCS: I.3.7 [Computer Graphics]: Three-Dimensional Graphics and Realism, Virtual Reality, Color, Shading, Shadowing, and Texture; I.3.3 [Computer Graphics]: Picture/Image Generation

\section{Introduction}

Projection-based appearances are important for many computer graphics applications. Altering the appearance of a physical scene with controlled digital projector light, or appearance editing (AE), offers a unique capacity to modify the visual appearance of objects outside a monitor's confines, allowing multiple simultaneous observers to enjoy the intuitive benefits of their human visual systems (e.g. depth perception, parallax, physical inspection). Previous AE sys- tems have virtually painted content onto objects [BRF01], have virtually restored cultural heritage [ALY08], and have increased the dynamic range of printed material to improve visualizations [BI08]. Other AE applications are discussed in Ref. [BIW08].

Achieving an $\mathrm{AE}$ requires that a physical surface (the scene) is capable of being visually modified to the colours of the desired target appearance (e.g. the ideal appearance sought by the observer). This means that a sufficient amount

\section{(c) 2011 The Authors}

Computer Graphics Forum (c) 2011 The Eurographics

Association and Blackwell Publishing Ltd. Published by

Blackwell Publishing, 9600 Garsington Road, Oxford OX4

2DQ, UK and 350 Main Street, Malden, MA 02148, USA. 
of light radiance from the projectors must be available to produce the target colour at each surface point in the scene. A compliant target appearance (or compliant appearance) is defined as an appearance whose colours can be achieved. In contrast, an incompliant appearance is an appearance that cannot be achieved due to colour and albedo constraints of the surface and projector light limitations. For example, a pure red surface albedo colour will have difficulty being edited to a pure blue target colour due to a lack of energy underneath the red colour's spectral response curve. In such a case, failing to produce the target colour may result in a misleading visualization. Short of altering the physical surface albedo or hardware setup, an incompliant appearance can only be made compliant by altering the target appearance. However, analysing the compliancy of a target appearance and minimally modifying it to be compliant has not been addressed in previous works. Rather, the target appearance has either been assumed to be compliant (e.g. [BRF01, RWLB01, JF07, BI08, OOD09]) or the target appearance's colours were naïvely enforced to be darker than the surface albedo's colour (e.g. [ALY08]), sacrificing brightness and possibly introducing unwanted colour changes due to gamut constraints. In general, the compliancy of a target appearance is dependent on the appearance's colours, the physical surface's albedo, and the amount of projector light radiance available from the setup.

\subsection{Key observations}

We make two key observations, which enable us to alter the initial target appearance to a perceptually similar compliant appearance (Figure 1). First, we decouple colour into its luminance and chrominance components and consider both components when modifying the target appearance. This allows our resulting compliant appearances to be brighter when compared to achieving compliancy only by reducing the target appearance's luminance (e.g. [ALY08]) and to be more chromatically accurate when compared to appearances generated by naïvely clamping the appearance's colours to the RGB range of the projectors. Although several colour spaces exist, we use CIELAB colour space (or, informally, Lab space) where $L^{*}$ represents a colour's lightness (a value related to relative luminance) and $\left(a^{*}, b^{*}\right)$ represent a colour's chrominance. Lab space was chosen for its ability to measure perceptual differences between colours with delta $E(\Delta E)$, which is identical to computing the Euclidean distance in a Cartesian coordinate system (in the 1976 definition; later definitions adjust the measurement to what is essentially a weighted Euclidean distance).

Secondly, because AE typically occurs in a dark room, no environment light exists to illuminate the scene's surrounding environment. Thus, in the absence of a reference colour from the environment, we exploit colour constancy [Wan95] to further maintain perceptual similarity. Colour constancy states that the perceived colour of an object remains constant despite changes in the spectrum of the illuminating light which in turn changes the spectrum of the light reflected from the object. Specifically, colour constancy is achieved when $\rho_{2}=R \rho_{1}$, where $R$ is a diagonal matrix describing colour ratios [Lan77] for all surface colours, and $\rho_{1}$ and $\rho_{2}$ are vectors containing the response of the three types of cones in our eyes under a first and a second illuminant. Colour constancy provides a stricter constraint than just luminance and chrominance similarity. In fact, preserving colour constancy suggests that the actual perceptual change may be smaller than the computed $\Delta E$.

\subsection{Method overview}

In a typical AE system, a three-dimensional (3D) model of the scene is first acquired and the projectors and camera are

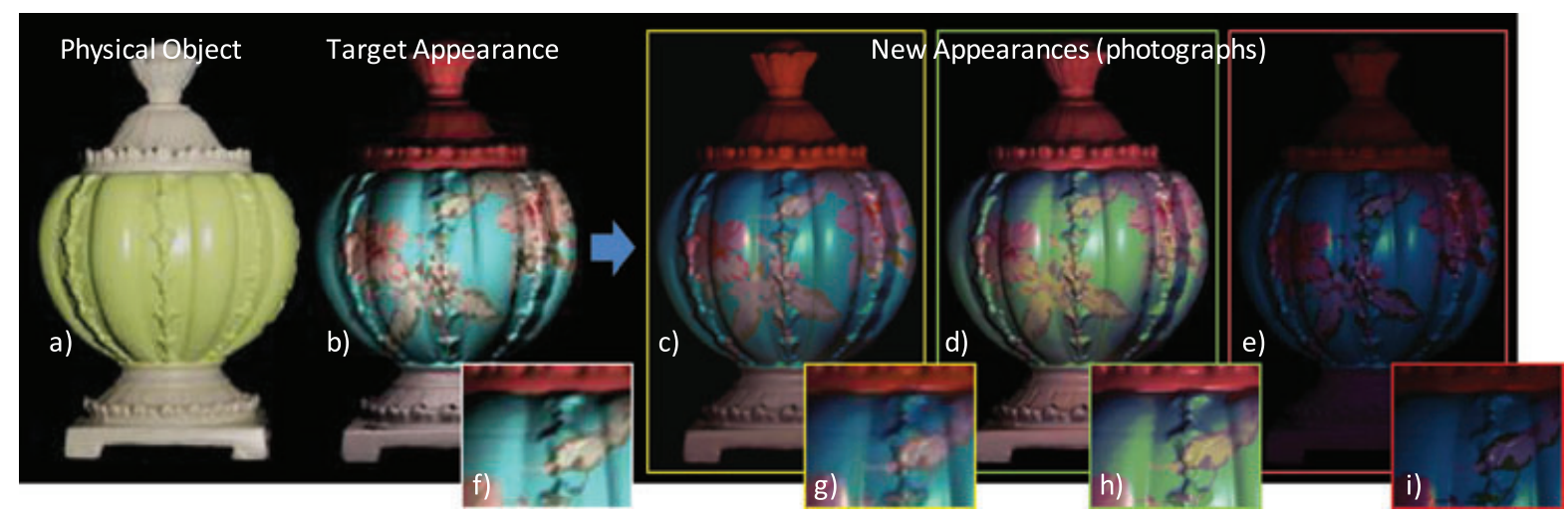

Figure 1: Compliant appearance editing. (a) Photo of physical object. (b) Incompliant target appearance (synthetic). (c) Photo of new appearance using our compliant target appearance. (d) Photo when naïvely applying the original target appearance. (e) Photo when reducing the original target appearance luminance until compliancy. $(f-i)$ Close ups. Naïvely using the original target appearance produces unexpected colour shifts. Reducing luminance obtains correct colours but a very dim look. Our approach yields the most perceptually similar appearance possible to the original target appearance. 


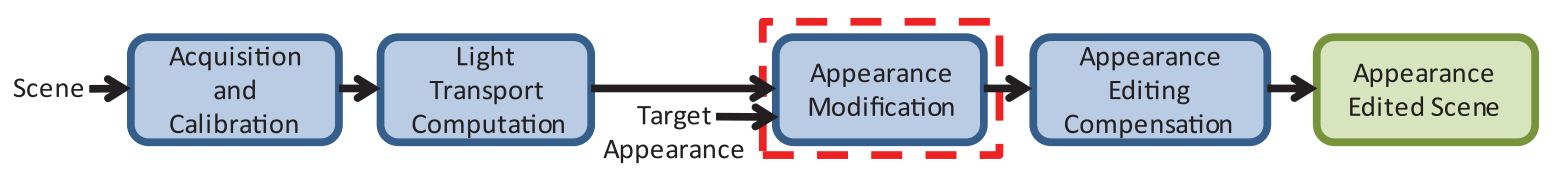

Figure 2: Modified appearance editing pipeline. Our method adds a step to a traditional appearance editing pipeline.

calibrated. Then, the projector-to-camera light transport is modelled. Finally, compensation images for each projector are generated which, when illuminating the scene, yield the target appearance. We add an appearance modification step to the pipeline (Figure 2), which seeks to minimally alter the target albedo colours and, as an approximation to colour constancy, to maintain their relative colour ratios.

The input to our method is a scene of one or more objects, the scene's physical surface albedo image $A_{s}\left(p_{i}\right)$, and a target appearance $T\left(p_{i}\right)=A_{t}\left(p_{i}\right) S\left(p_{i}\right)$. $A_{s}$ and $T$ are defined for all surface points $p_{i}$ visible from an observer's viewpoint (typically a camera). $A_{s}$ and $A_{t}$ are flat coloured albedo images; $A_{s}$ contains each surface point's surface albedo under maximal projector illumination, and $A_{t}$ contains the target colours with no shading effects. $S$ is the target appearance-shading model (e.g. diffuse, specular). The output of our method is a compliant target appearance $T_{c}\left(p_{i}\right)=A_{c}\left(p_{i}\right) S\left(p_{i}\right)$ where $A_{c}$ is a modified compliant albedo image and $S$ is the same target shading model.

Our appearance modification method first partitions the scene's surfaces into appearance editing patches which are used to organize contiguous surface points with nearly the same colour in both $A_{s}$ and $A_{t}$. Patch creation is motivated by the fact that all points in a patch require the same change in colour during appearance optimization. Our appearance optimization minimally alters the patches' albedo colours $A_{t}$ and produces the compliant albedo colours $A_{c}$ and thus the compliant appearance $T_{c}$. When altering $A_{t}$ 's colours, we balance the preservation of three cues critical to perceptual similarity. First, maintaining the chrominance of $A_{t}$ 's colours is important because uncontrolled chrominance change breaks colour constancy and results in a dissimilar compliant appearance. Secondly, although luminance is invariably lost in $A_{t}$ to achieve compliancy, the loss should be kept small. Finally, maintaining the ratios of $A_{t}$ 's colours preserves colour constancy thus further reducing the perceived difference between $T$ and the modified $T_{c}$.

We have implemented a prototype AE system, which supports imparting new compliant appearances onto arbitrary objects. We assume our objects contain diffuse surfaces with no indirect illumination from projector light (e.g. interreflections). Further, although the imparted appearances can have colour gradients due to shading and illumination, we assume that both $A_{s}$ and $A_{t}$ consist of patches of nearly constant colour. Intuitively, this means that under ambient light, the physical object and the target appearance should each consist of contiguous regions of similarly coloured points.
Such an assumption is well suited for many man-made and painted objects. In our results, we compare our compliant appearances to the original, incompliant target appearances ignoring compliancy issues as well as to appearances achieving compliancy by only reducing the appearance's luminance. $\Delta E$ is used to measure perceptual similarity. In all cases, our method shows improved visual quality and perceptual similarity.

\section{Previous Work}

Most research in appearance editing has assumed the use of compliant target appearances (e.g. [RWLB01, BRF01, BI08, OOD09]) and has instead focused on computing compensation images to achieve the desired appearance. Some of these works mitigate the appearance compliancy issue by dealing strictly with white or near-white surfaces, which maximize the gamut of compliant colours possible (e.g. [RWLB01, BRF01, OOD09]). However, there is no guarantee that an appearance is compliant.

Radiometric calibration is used in $\mathrm{AE}$ as a means to increase the accuracy of reproducing colours (e.g. [NPGB03, GPNB04, WB07, GB08, SLM10]). However, radiometric calibration cannot compensate for strong colour changes where an insufficient amount of projector light radiance makes achieving a target colour fundamentally impossible. As a result, these incompliant colours are clipped in colour space, resulting in undesirable and clearly visible colour shifts. In this situation, one can increase the available projector light radiance at hard-to-edit areas by placing the projectors more head-on to these areas (e.g. [LAM10]), but there is still no guarantee that a sufficient amount of light radiance will be available to achieve the desired AE. The only way to guarantee adequate projector light radiance is by adding additional projectors to the AE setup. Because additional projectors may not always be practical, we instead opt to modify the target appearance to achieve a compliant AE.

Aliaga et al. [ALY08] consider an appearance's compliancy by preferring target point colours to be darker than surface albedo colours. Only considering luminance fails to consider the full spectral reflectance of the surface. Suppose a physical surface with a spectral reflectance curve containing high values near the red frequencies is to be edited to a colour with high values in the blue frequencies, but lower luminance. Although the luminance is reduced in this colour change (and thus the change is luminance compliant), the colour change is not possible due to a lack of energy in the blue wavelengths of the surface's spectral reflectance curve. 
Thus, although the compliancy of the appearance may be improved, it is not guaranteed.

Achieving accurate colours has been addressed for multi-projector display systems. Because a projector's colour properties change over space (due to overlapping projectors, vignetting and intra-image variations due to projector hardware), a target image may look different than expected or show noticeable seams. To achieve a smooth appearance across the projected imagery, the projected images are modified (e.g., altering the brightness [MS05] or the colour gamut [SLMG09]). Sajadi et al. [SLMG09] assure a smoothly changing colour gamut between projected imagery. However, the projected imagery is not guaranteed to be compliant. Instead, the projections appear on standard white or back-projection screens to minimize the chance and impact of incompliancy. Further, the difference of the desired appearance and the projected appearance is not minimized. Thus, the final colours obtained may be needlessly different from the target colours.

Our work is also related to content-dependent gamut mapping. Such methods consider an image's colour data (e.g. [Bra99, HOK99, BF99]) and account for the spatial relationship between an image's colours (e.g. [BDEW01, KSES05, ZS07]) to map out-of-gamut image colours to in-gamut display device colours, often assuming the gamuts are relatively similar. We consider both an appearance's colours and the spatial relationship between the colours to map a set of incompliant colours to a set of compliant colours. Three factors unique to our AE objectives differentiate our work from typical gamut mapping methods: (i) each surface albedo patch colour defines a target gamut of appearance compliant colours based on its colour, geometry, and the available projector light radiance; (ii) target colours which span multiple surface albedo colours must remain constant across multiple appearance editing patches despite differences in the underlying surface albedo and while constraining the direction of colour shifts of the target colours to the compliant target gamut and (iii) the balance of maintaining the spatial ratios of the target colours with minimizing the change to the target colours further constrains the direction of the colour shifts of the target colours to the compliant target gamut.

\section{Colour Compliancy}

A key concept in our appearance modification method is ensuring the target appearance is compliant. Thus, we begin the description of our approach by presenting a definition of colour compliancy and describing a technique for measuring it first in RGB space and then in Lab space.

\subsection{Definition}

A target colour is compliant if the amount of projector light radiance available is sufficient to produce the target colour for a given surface albedo colour at a particular surface point. Previous AE systems work with colour as a point in RGB space (e.g. [RWLB01, GPNB04, ALY08, GB08]) where each colour channel encapsulates both perceived chrominance and perceived luminance. Thus, luminance increases monotonically within each RGB channel, and a simple approximation to ensuring compliancy is to alter each target colour channel so that each channel value is less than the amount of available projector light radiance. However, because chrominance is not constant when varying any of the RGB colour channels, achieving compliancy using this naïve methodology implies an obfuscated mix of changing perceived chrominance and perceived luminance.

We define colours in Lab space to decouple a colour's luminance and chrominance to separately and minimally alter these components. Thus, achieving compliancy is no longer verifying that each target colour's channels are less than the available projector light radiance. Instead, a new chrominance to luminance mapping must be created for each surface point $p_{\mathrm{i}}$ to determine whether or not a particular target colour is compliant. To create this mapping, we use the compliancy definition in RGB space (Section 3.2) as a building block for the more accurate definition in Lab space (Section 3.3).

\subsection{Light availability and compliancy in RGB space}

In RGB space, the total amount of radiance available $L$ in a given $M$ projector setup at a particular surface point $p_{i}$ is defined by the $3 \mathrm{D}$ vector

$$
L\left(p_{i}\right)=b_{a}+A_{g}\left(p_{i}\right) \sum_{i}^{M}\left(b_{j}+\frac{L_{\max }\left(n_{i}-l_{j}\right)}{d_{I j}^{2}}\right),
$$

where $\left(n_{i}-l_{j}\right)$ represents the angular attenuation due to the projector-surface point orientation (assuming a diffuse surface, $l_{j}$ is the directional vector to projector $j$ and $L_{\max }$ is the maximum luminance available from a projector), $1 / d_{i j}^{2}$ is the distance attenuation from projector $j$ to surface point $p_{i}$, $b_{\mathrm{a}}$ is a vector corresponding to ambient black-level lighting and $b_{j}$ is a vector of the black-level colour of projector $j$.

For simplicity, we approximate $L$ by assuming the values for $b_{\mathrm{a}}$ and all $b_{j}$ 's are small and thus negligible; radiometric calibration should compensate for their contributions as well (unless the target colour is dimmer than the black level lighting, making it fundamentally impossible to achieve). Thus, the approximate amount of radiance available $L$ at $p_{i}$ is given by the RGB vector

$$
\widehat{L}\left(p_{i}\right)=A_{s}\left(p_{i}\right) \sum_{i}^{M} \frac{L_{\max }\left(n_{i}-l_{j}\right)}{d_{i j}{ }^{2}} .
$$

A target appearance is compliant if the amount of available projector light radiance is greater than or equal to each

(c) 2011 The Authors Computer Graphics Forum (c) 2011 The Eurographics Association and Blackwell Publishing Ltd. 
channel of the target colour $T\left(p_{i}\right)$ in each RGB channel:

$$
\widehat{L}\left(p_{i}\right) \geq A_{t}\left(p_{i}\right) S\left(p_{i}\right)
$$

Because our colour optimization-described in Section 5 -modifies target albedo colours in $A_{t}$, we isolate $A_{t}\left(p_{i}\right)$ by dividing by the scalar $S\left(p_{i}\right)$ to yield an equivalent vector of inequalities which expresses per-RGB channel colour compliancy. Along with expanding $L$, this yields

$$
A_{s}\left(p_{i}\right) \times \underbrace{\frac{1}{S\left(p_{i}\right)} \sum_{j}^{M} \frac{L_{\max }\left(n_{i}-l_{j}\right)}{d_{i j}{ }^{2}}}_{\text {attenuation }} \geq A_{t}\left(p_{i}\right) .
$$

For the case when $S\left(p_{i}\right)=0$ (e.g. a point back-facing the projectors), the attenuation is irrelevant because the point will always be compliant (i.e. assuming no ambient blacklevel light, perfect black is always achievable).

\subsection{Light availability and compliancy in lab space}

Our solution for creating the chrominance to luminance mapping in Lab space is a compliancy heightfield $H_{i}$ specific to each surface point $p_{i}$, its albedo, and its amount of available projector light radiance (Figure 3 ; the $b^{*}$ axis is omitted for simplicity in the figure). This heightfield $L^{n}=H_{i}\left(a^{*}, b^{*}\right)$ takes $p_{i}$ 's surface albedo into account to describe the amount of luminance available at $p_{i}$ for a desired target colour chrominance given the current projector setup. In other words, $L^{n}$ defines the maximum lightness for a given target colour's chrominance $\left(a^{*}, b^{*}\right)$ which can be achieved at $p_{i}$ in the current $\mathrm{AE}$ setup [i.e. the left-hand side of Equation 4 for a chrominance $\left.\left(a^{*}, b^{*}\right)\right]$.

\subsubsection{Compliancy heightfield construction}

The heightfield $H_{i}$ for a point $p_{i}$ is constructed by sampling the $a^{*}$ and $b^{*}$ axes and computing $L^{n}$ for each sampled ( $a^{*}$, $\left.b^{*}\right)$. Both $a^{*}$ and $b^{*}$ are sampled in their full ranges $[-128$, 128], each at $s_{h}$ unit intervals. In practice, we found $s_{h}=4$ to balance computational efficiency and modelling accuracy. A sparser sampling of $a^{*}$ and $b^{*}$ produces a less accurate height- field, and a denser sampling of $a^{*}$ and $b^{*}$ makes computing the heightfield functions (Section 3.3.2) less efficient. For each $\left(a^{*}, b^{*}\right)$ sample, the maximum value for $L^{n}$ is found such that the corresponding RGB vector is compliant given the corresponding surface albedo colour $A_{s}\left(p_{i}\right)$. This maximum luminance value is computed by initializing $L^{n}$ to 100 (the maximum $L^{*}$ value) and iteratively decreasing $L^{n}$ until compliancy is attained. Compliancy is determined by converting $\left(L^{n}, a^{*}, b^{*}\right)$ to RGB space and ensuring that the resulting RGB colour is compliant. Now, $H_{i}$ satisfies $A_{s}\left(p_{i}\right) \geq A_{t}\left(p_{i}\right)$.

To account for attenuation due to the projector-object relationship and $S$, we scale each $L^{n}$ by the attenuation factor in Equation (4) to achieve the final heightfield $H_{i}$. The heightfield surface now represents a compliancy threshold with Lab space colours below the surface being compliant and colours above the surface being incompliant.

\subsubsection{Compliancy heightfield functions}

Next, we describe two compliancy functions $C_{i}\left(t_{i}\right)$ and $D_{i}\left(t_{i}\right)$ to extract information about a target colour $T\left(p_{i}\right)$ using $p_{i}$ 's compliancy heightfield $H_{i}$. Because $S\left(p_{i}\right)$ is incorporated in $H_{i}$, both compliancy functions use $t_{i}=A_{t}\left(p_{i}\right)$, the target albedo colour, as the colour input.

$C_{i}\left(t_{i}\right)$ measures the compliancy of target colour $T\left(p_{i}\right)$ at surface point $p_{i}$ with compliancy heightfield $H_{i}$ and is defined as

$$
C_{i}\left(t_{i}\right)=H_{i}\left(t_{i}\left[a^{*}\right], t_{i}\left[b^{*}\right]\right)-t_{i}\left[L^{*}\right] .
$$

This function yields a scalar value where $C_{i}$ 's sign indicates compliancy or incompliancy and $C_{i}$ 's magnitude represents the degree of compliancy or incompliancy. To compute $C_{i}\left(t_{i}\right)$, the distance in $L^{*}$ between $t_{\mathrm{i}}$ and $H_{i}$ is measured. Intuitively, if $t_{i}\left[L^{*}\right]>H_{i}\left(t_{i}\left[a^{*}\right], t_{i}\left[b^{*}\right]\right)$ (i.e., comparing the luminance of $t_{i}$ and $\left.H_{i}\left(t_{i}\left[a^{*}\right], t_{i}\left[b^{*}\right]\right)\right)$, then the target colour is incompliant and $C_{i}\left(t_{i}\right)<0$, else it is compliant and $C_{i}\left(t_{i}\right) \geq 0$ (Figure 3).

$D_{i}\left(t_{i}\right)$ measures the perceptual distance between a target colour and its closest compliant colour. This function also yields a scalar value, which is the minimum perceptual

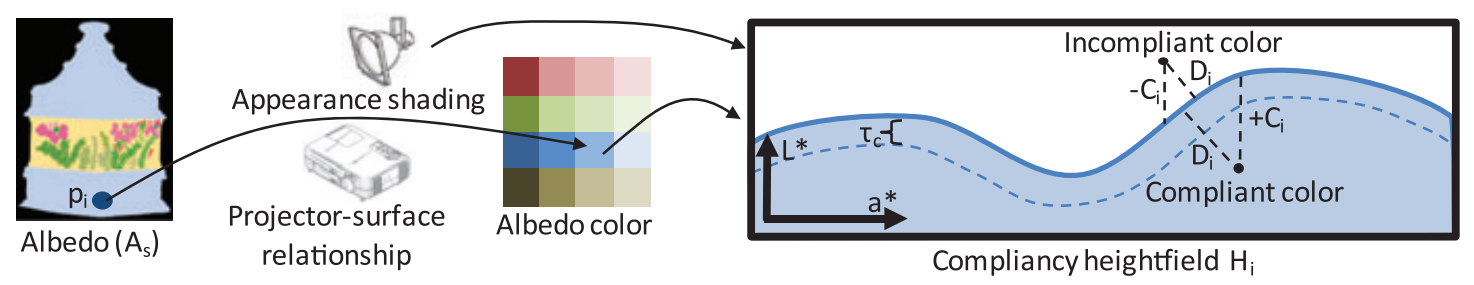

Figure 3: Compliancy heightfield. The compliancy heightfield $H_{i}$, defined in Lab colour space, indicates the maximum luminance for any desired chrominance at surface point $p_{i}$. (Note: the heightfield is a $2 D$ manifold but here only $L^{*}$ and $a^{*}$ axes are shown.) Incompliant colours are above the heightfield surface with $c_{i}<0$, and compliant colours are beneath. 
distance for an incompliant colour to become compliant. To compute $D_{i}$, a weighted distance from $t_{i}$ to the closest colour on the compliancy threshold of $H_{i}$ is used (Figure 3). The weights $\left(w_{L^{*}}, w_{a^{*}}, w_{b^{*}}\right)$ enable different importance to be given to luminance and to each of the chrominance axes (e.g., if $w_{L^{*}} w_{a^{*}}, w_{b^{*}}$, the $L^{*}$ axis is the most important to preserve, and changes in luminance are penalized, which is conmensurate with human perception properties) and are used to control the appearance optimization process. Although the above is conceptually clear, in our implementation, we use the weights to scale each component of $t_{i}$ as well as all sampled points on $H_{i}$, yielding values $t_{i}$ and $H_{i}$. Then, $D_{i}$ is defined as a standard Euclidean distance between $t_{i}$ and the nearest surface point on $H_{i}$.

\section{Appearance Editing Patches}

The next step is to create AE patches over the scene's surfaces to succinctly formulate compliancy equations. Given a scene's surface albedo image and target albedo image, patches are created by dividing the scene's surfaces into regions containing a nearly constant surface albedo and a nearly constant target albedo (Figure 4). With a per-projector pixel radiometrically calibrated system, the albedos need not be exactly constant but only sufficiently similar so that all points within a patch can be assumed to be changeable in a similar manner. Overall, our partitioning strategy helps reduce and avoid introducing unwanted sharp colour boundaries within each patch.

\subsection{Surface partitioning}

The scene's surfaces are partitioned into patches by grouping contiguous pixels sharing a similar surface albedo colour and the same target albedo colour. Using the surface albedo image $A_{s}$ and the target albedo image $A_{t}$, pixels in each of $A_{s}$ and $A_{t}$ are grouped into sets of contiguous similar-colour patches, called surface patches and target patches, respectively. Colours in $A_{s}$ are computed with colour segmentation

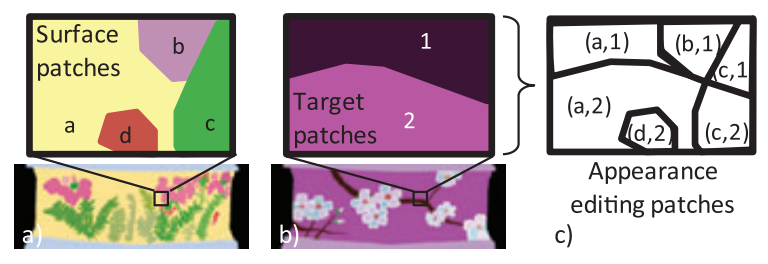

Figure 4: Appearance editing patches. (a) Using the surface albedo, surface patches are created. (b) Similarly, using the target albedo, target patches are created. (c) The appearance editing patches used during appearance optimization group contiguous pixels with the same surface patch colour and same target patch colour. (e.g. mean shift segmentation [CM02]) on a photograph of the scene under ambient light (in practice, a photo editing tool is used afterwards to handle poorly segmented areas). $A_{t}$ is designed with constant colour patches for simplicity. Each scene pixel is thus part of a single surface patch and is also part of a single target patch. AE patches are then formed by grouping contiguous pixels sharing the same surface patch and target patch.

\subsection{Selecting a representative patch point}

For each AE patch $k \in[1, p]$, a single representative surface point $r_{k}$ is identified to characterize the patch in a formulation of compliancy for the entire scene. Because the captured model has a very large number of (scanned) surface points, using all points when formulating patch compliancy equations is prohibitive. By using a single surface point to represent a patch's compliancy across its surface, careful selection of $r_{k}$ is crucial for a robust appearance optimization process. Naïvely, one may wish to represent an entire patch with a geometric averaging of the patch's surface points (e.g. average the positions and normals of the patch). Although such a virtual point may seem to be a reasonable estimation of a patch's physical properties, it does not reflect the compliancy of any actual point on the patch. Further, it could misrepresent compliancy and hence skew the colour optimization. Instead, we analyse the degree of compliancy [as measured by $C\left(p_{i}\right)$ ] of many points $p_{i}$ in patch $k$ and choose as $r_{k}$ a point whose compliancy is near the mean $C\left(p_{i}\right)$ value of the patch. An analysis of the impact of selecting different points for $r_{k}$ 's is presented in the results (Section 6).

\section{Appearance Optimization}

Appearance optimization uses all patches to iteratively alter the target albedo colours $A_{t}\left(r_{k}\right)$ to produce a set of compliant albedo colours $A_{c}\left(r_{k}\right)$ (and thus $T_{c}$ ). The overall relationship between a change in colour, a change in the colour ratio between patches, and a change in compliancy is non-linear. Our methodology uses a gradient descent based optimization to iteratively find a colour-compliant solution that balances colour changes and colour ratio changes. During each iteration, a set of linear equations is used to balance several heuristics described in this section, and a small colour shift is computed in the direction of steepest descent (over $D_{k}$ ) towards compliancy for each patch. The patch colours are changed until each $r_{k}$ lies below $H_{k}$ 's surface (or a maximum number of iterations is reached).

\subsection{Colour shifting methodology}

We first define some notation: (i) $c_{\mathrm{km}}$ refers to the current target colour of patch $k$ during iteration $m$ (i.e., $c_{k 0}=A_{t}\left(r_{k}\right)$ is the initial target colour and $c_{k m}=A_{c}\left(r_{k}\right)$ for some, value of $m$ ); (ii) $t_{k m}$ refers to the desired more compliant patch colour 
during iteration $m$ and (iii) $s_{k m}=\left\{S_{k m l^{*}}, S_{k m a^{*}}, S_{k m b^{*}}\right\}$ refers to the colour shift for patch $k$ during iteration $m$, computed via an optimization. If the amount of change in patch colours and in patch colour ratios is ignored, compliant colours can be calculated for each $t_{k m}$ in one iteration using the trivial equations

$$
\begin{gathered}
s_{k m}=t_{k m}-c_{k m}, \\
c_{k(m+I)}=c_{k m}+s_{k m} .
\end{gathered}
$$

However, to reach a compliant appearance perceptually similar to $A_{t}$, the simultaneous effect of all colour shifts are considered in a linear optimization. Thus, several iterations are needed to converge to a balanced solution.

The gradient $g_{k m}$ of each $D_{k}\left(c_{k m}\right)$ is computed using finite differences, and the next iteration's target patch colours are defined as

$$
t_{k m}=\left\{\begin{array}{l}
c_{k m}-d g_{k m} \text { if patch } k \text { is incompliant, } \\
c_{k m}+d g_{k m} \text { if patch } k \text { is barely compliant, }
\end{array}\right.
$$

where $d$ is a user-selected value (e.g. 0.05) controlling the rate of change of $c_{k m}$. For incompliant patches $\left[C_{k}\left(c_{k m}\right)<0\right], t_{k m}$ is moved towards compliancy. For barely compliant patches $\left(0 \leq C_{k}\left(c_{k m}\right) \leq \tau_{c}, t_{k m}\right.$ is moved away from $H_{k}$ 's compliancy threshold towards a more compliant colour. Moving barely compliant patches reduces patches from oscillating in and out of compliancy provided that $d_{g k m}<\tau_{c}$. Colour constancy is preserved by allowing more compliant patch colours $\left(c_{k}\left(c_{k m}\right)>\tau_{c}\right)$ to move freely because they are not at risk of becoming incompliant in a single iteration. During each iteration, the set of incompliant and barely compliant patches is updated, and colour shifts $\mathrm{s}_{k m}$ are computed via the linear optimization.

\subsection{Patch equations}

To simultaneously solve for all $s_{k m}$ 's during an iteration of the colour shifting process, a linear system consisting of two types of equations is optimized. The equations aim to have each $c_{k m}$ reach $t_{k m}$ while encouraging a desired balanced change in colour and change in colour ratios.

\subsubsection{Patch colour equations}

Patch colour equations focus on finding the most perceptually similar appearance compliant colour for each AE patch. For a patch $k$ during iteration $m$, three straightforward linear equations are defined by using Equation (6) for each colour channel of incompliant and barely compliant patches. By using equations for all three Lab channels and the weights $\left(w_{L^{*}}, w_{a^{*}}, w_{b^{*}}\right)$ within $D_{k}$, the colour shifts are steered to the closest compliant colour relative to a weighted importance metric.

\subsubsection{Patch ratio equations}

Patch pair equations ensure colour constancy in the modified appearance by requiring that the colour ratio between two AE patches remains similar to the original target appearance colour ratios. For all pairs of patches $k_{1}$ and $k_{2}$, $R_{k_{1} k_{2}}=c_{k_{1} 0} / c_{k_{2} 0}$ is computed as the original per-channel colour ratio in Lab space. Then, during an iteration $m$, the colour ratio of patch $k_{1}$ to $k_{2}$ is constrained to remain the same after a colour shift by $s_{k_{1} m}$ and $s_{k_{2} m}$; namely,

$$
c_{k_{1} m}+s_{k_{1} m}=R_{k_{1} k_{2}}\left(c_{k_{2} m}+s_{k_{2} m}\right) \text {. }
$$

Perceptually, the importance amongst different patch pair ratio equations is also dependent on the physical distance between the two patches. Thus, a multiplicative weight $w_{k_{l} k_{2}}$ is added to each patch pair equation. Further, because $a^{*}$ and $b^{*}$ range from $[-128,128]$, their values are shifted to $[0,256]$ when calculating the ratios. After some algebraic rearrangement, the patch ratio equations become

$$
w_{k_{1} k_{2}}\left(s_{k_{1 \mathrm{~m}}}-R_{k_{1} k_{1}} s_{k_{1} m}\right)=w_{k_{1} k_{2}}\left(R_{k_{1} k_{2} C_{k_{2}} m}-c_{k_{1} m}\right),
$$

where $w_{k_{1} k_{2}}=d_{k_{1} k_{2}} / d_{\max }, d_{k_{1} k_{2}}$ is the distance between patches $k_{1}$ and $k_{2}$, and $d_{\max }$ is the maximum distance between any two patches.

The number of patch ratio equations gets quite large for scenes with complex surface albedos and target appearances. Hence, patch pairs where $d_{k_{l} k_{2} \geq} \bar{d}_{k_{l} k_{j}}+n_{d} \sigma_{d}$ are ignored, where $\bar{d}_{k_{l} k_{j}}$ is the average patch pair distance, $\sigma_{d}$ is the standard deviation of patch pair distances, and $n_{d}$ is a user-defined threshold defining the magnitude of patch pair distances to ignore.

\subsection{Iterative optimization}

Using these equations, a solution to the over-constrained linear system is computed using linear least squares. For further control, a weight $\alpha \in[0,1]$ is provided to balance the importance of patch colour equations to patch pair equations. Given $P_{c}$ patch colour equations, $P_{p}$ patch pair equations and a total of $P_{t}=P_{\mathrm{c}}+P_{p}$ equations, this results in the weights $\alpha\left(p_{c} / p_{t}\right)$ and $(1-\alpha)\left(p_{p} / p_{t}\right)$ to be multiplied into Equations (6) and (10), respectively.

The iterative optimization is terminated when: (i) all $\mathrm{AE}$ patch colours result in a compliant target appearance; (ii) the magnitude of the colour shift is underneath a threshold $\tau_{p}$; in theory a $\Delta E \approx 2.3$ is considered a just noticeable difference [Sha03], however this value is derived from an unscaled Labspace; because our domain is scaled by $\left(w_{L^{*}}, w_{a^{*}}, w_{b^{*}}\right)$ small iterative steps still may add up to a noticeable colour shift, 
thus we choose the conservative value of $\tau_{p}=0.02$ or (iii) a maximum number of iterations is reached.

\section{Results and Discussion}

Our system acquires a model, calibrates the projectors and camera, and captures projector-to-camera light transport similar to [ALY08]. Radiometric calibration is performed using [SLM10] or [ALY08]. Then, a compliant appearance $A_{c}$ is computed for a given target appearance. For simple scenes, $A_{c}$ is computed in a few minutes using all patch ratio equations $\left(n_{d}=\infty\right)$. For complex scenes, we set $n_{d}=-1$ to limit computation time to about an hour. Weights for $D_{i}$ are set to $w_{L^{*}}=5, w_{a^{*}}=2$ and $w_{b^{*}}=1$ to discourage reducing luminance and encourage shifting in $b^{*}$ rather than $a^{*}$. Shifting along the blue-yellow opponent channel was preferred because the human visual system is believed to be less sensitive to shifts along this axis [LPA07]—because the sun is yellow/white and the sky is blue. Hence, a shift in $b^{*}$ is likely to be interpreted as a change in the illuminant (conveying colour constancy) whereas a shift in $a^{*}$ is likely to be perceived as a change in the surface. The flexibility in choosing weights enables our framework to be used in other applications where shifts in other channels may be preferred.

Table 1 summarizes our experimental scenes and shows the benefit of using our method's compliant appearances as compared to using naïve appearances generated by reducing the original target appearance's luminance until compliancy is attained. For each scene, the two resulting appearances' average lightness (average $L^{*}$ value) and average $\Delta E$ (1976 definition, when compared against the original target appearance) over the entire AE were computed. Our method's appearances yield a higher average $L^{*}$ and a lower average $\Delta E$. This shows that considering both chrominance and luminance together can produce compliant appearances which are both brighter and more perceptually similar to the target appearance when compared to appearances generated by

Table 1: Scene $\Delta E$. Average perceptual differences measured by $\Delta E$ across each scene. We compare the target appearance against both our method's compliant appearance and another compliant appearance naively generated by only reducing the appearance's luminance until compliancy is achieved. For all scenes, our compliant appearance is brighter and more perceptually similar to the target

\begin{tabular}{lrrrrr}
\hline & \multicolumn{2}{c}{ Our method } & & \multicolumn{2}{c}{ Lum reduction } \\
\cline { 2 - 3 } \cline { 6 - 6 } Scene & \multicolumn{1}{c}{$L^{*}$} & \multicolumn{1}{c}{$\Delta E$} & & \multicolumn{1}{c}{$L^{*}$} & \multicolumn{1}{c}{$\Delta E$} \\
\hline Ped-checker (Fig 5) & 24.283 & 30.123 & & 9.144 & 47.497 \\
Ped argyle (Fig 8) & 14.818 & 3.970 & & 14.319 & 24.533 \\
Pumpkin (Fig 1) & 32.556 & 18.471 & & 11.693 & 38.071 \\
House (Fig 10) & 15.878 & 43.348 & & 2.933 & 51.143 \\
Vases (Fig 9) & 23.735 & 18.403 & & 16.484 & 25.939 \\
\hline
\end{tabular}

only considering luminance. The absolute $\Delta E$ values shown are not very relevant because the intensity level of the (synthetic) original target appearance is arbitrary-a very bright original target appearance may fundamentally require a drastic change given a limited amount of projector light radiance. Instead, the relative change in $\Delta E$ is more important because it indicates the amount of perceptual change required by each approach to reach compliancy. Moreover, because our method attempts to preserve colour constancy, our resulting appearances may be even more perceptually similar than the calculated $\Delta E$ values. Hence, although our method reports relatively large $\Delta E$ values, the perceptual error might be significantly less during an observation. Regardless, although some resulting modified appearances may not seem perceptually equivalent (and numerically such due to some relatively large $\Delta E$ values), the modified appearances nonetheless are generally perceptually similar enough to convey the same information of the original target appearances.

Figure 5 contains an exemplary appearance edit and some comparisons. The physical object and incompliant target appearance are shown in Figures 5a and b, and photos of the new appearances are shown in Figures $5 \mathrm{c}$ and e. As demonstrated by Figures $5 \mathrm{f}$ and $\mathrm{g}$, a naïve use of the target appearance using a system similar to [ALY08] and no appearance modification results in large changes in colour-a colour shift occurred. Figures $5 \mathrm{~h}$ and $\mathrm{i}$ show that reducing the target appearance's luminance achieves compliancy but an overall dim look. These visualizations are computed using a perpixel $\Delta E$ strictly over the chrominance and luminance aspects of the colours, respectively. Our appearance balances this trade-off. Close-ups are provided in Figures 5j-m.

In Figure 6, we analyse the effect of $\alpha$ which controls the importance between individual patch colours and patch-topatch colour ratios. Figure 6(a) shows a portion of a target appearance, and Figure 6(b) shows a photograph sequence of compliant AEs using $\alpha$ values in the range [0,1] (the full appearance is shown in Figure 1). Appearances using low $\alpha$ show reduced luminance. In contrast, appearances using high $\alpha$ appear brighter but with obvious chromatic errors: colour details are washed out in the bottom half of the shown appearance, and there is inappropriate relative brighting of the petal near the middle of the image. Figure 6(c) shows a quantitative comparison. $\Delta E$ represents colour difference using a scalar; however, the particular selection of weights for the luminance and chrominance components can significantly alter the resulting value. Thus, we decompose $\Delta \mathrm{E}$ into a normalized luminance error and a normalized chrominance error. The red curve shows the luminance error; conmensurate with the images, the brighter appearances at larger $\alpha$ values have a smaller error. The blue curve shows the chrominance error with a clear U-shape curve having a minimum near $\alpha=0.66$. We typically use $\alpha \in[0.5,0.66]$.

Figure 7 demonstrates the effect of selecting different representative patch points $r_{k}$ to achieve the appearance in 


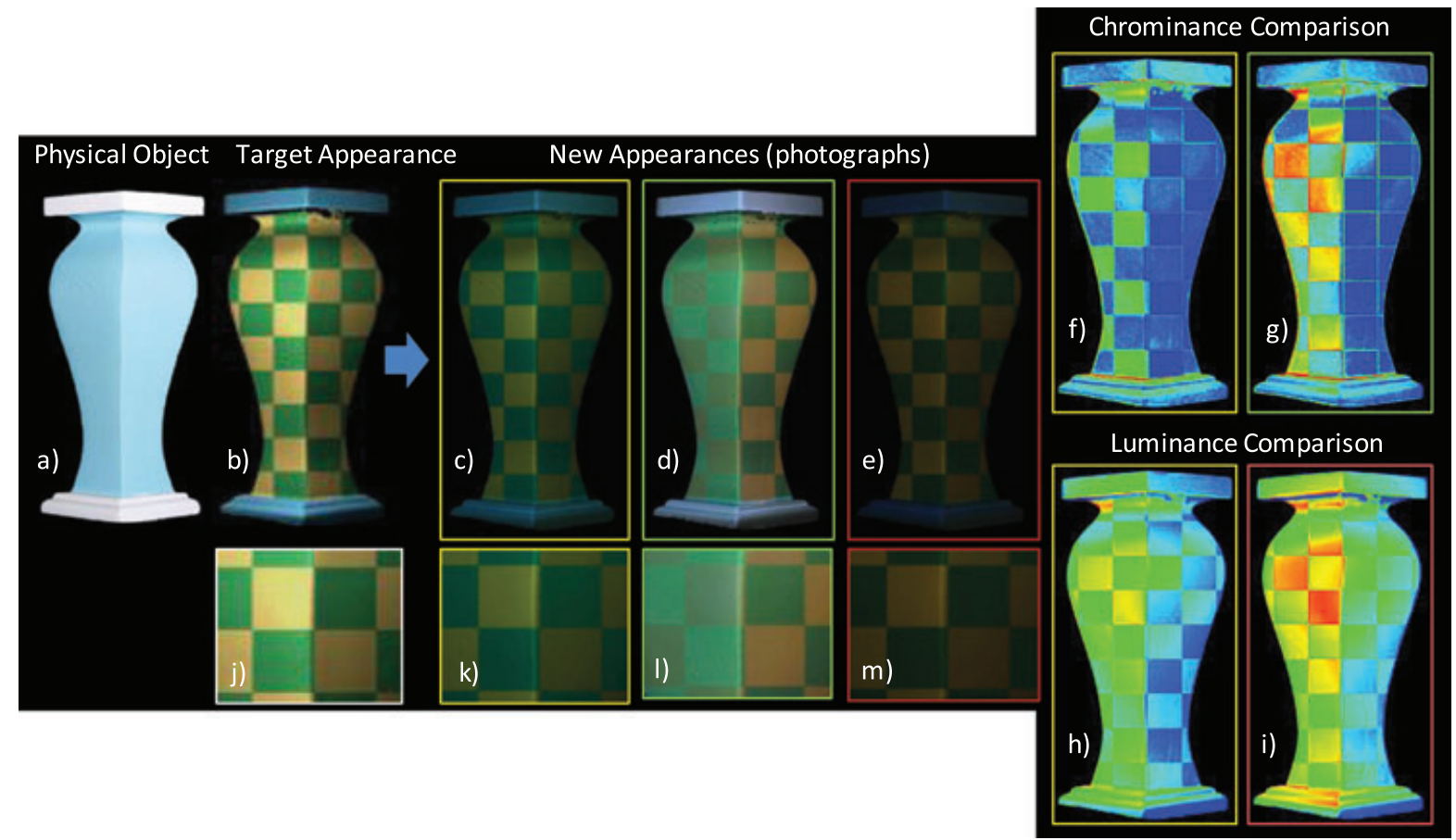

Figure 5: Example pipeline. (a) Photo of physical object. (b) Image of incompliant target appearance. (c) Photo of AE using our compliant appearance image. (d) Photo of AE naïvely using target appearance. (e) Photo of AE when reducing luminance until compliancy. ( $f$ and $g$ ) Colourmap showing chromatic difference of $(b)$ against $(c)$ and $(d)$ where blue-cyan-green-yellow-red maps to increasing difference. $(h-i)$ Colourmap showing luminance difference of $(b)$ against $(c)$ and $(e)$. Results in $(f)$ and $(h)$ indicate better perceived similarity of our solution to the target. $(j-m)$ Close-up views.

a)

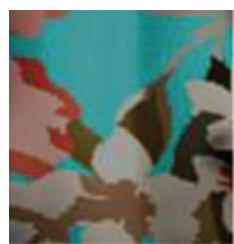

b)
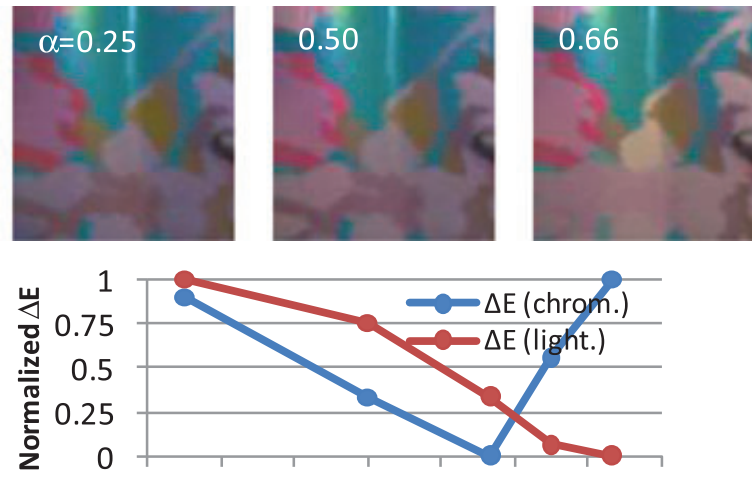

c)

$$
\begin{array}{llllllll}
0.2 & 0.3 & 0.4 & 0.5 & 0.6 & 0.7 & 0.8 & 0.9
\end{array}
$$

Figure 6: Analysis of colour change vs. patch-ratio change. (a) Image of target appearance. (b) Photos of a compliant AE using various $\alpha$ values. (c) Graph showing the normalized luminance and chrominance components of $\Delta E$ for the shown images. Low $\alpha$ values produce dim appearances but have reasonable chromatic accuracy. High $\alpha$ values produce brighter appearances but have noticeable chromatic errors.

Figure 1. For a patch $k$, we calculate the mean patch compliancy value $\bar{C}_{k}$ and its standard deviation $\sigma_{C_{k}}$ over all patch points and select $\tau_{k}$ based on these values. Selecting a point with an initial compliancy value less than $\bar{C}_{k}$ (e.g. $\bar{C}_{k}-\sigma_{C_{k}}$ or $\bar{C}_{k}-2 \sigma_{C_{k}}$ ) encourages an aggressive colour shift towards compliant colours at the expense of luminance (Figure $7 \mathrm{a}$ and b). In contrast, selecting a point with an initial compliancy value greater than $\bar{C}$ (e.g. $\bar{C}_{k}+\sigma_{C_{k}}$ or $\bar{C}_{k}+\sigma_{C_{k}}$ ) encourages 
a)

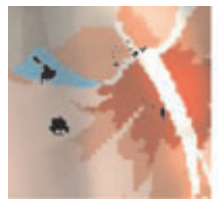

b)

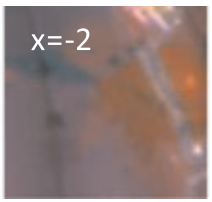

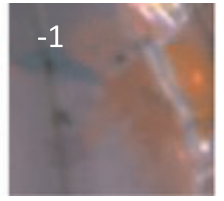

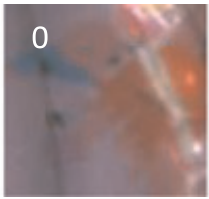

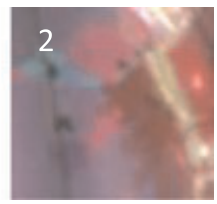

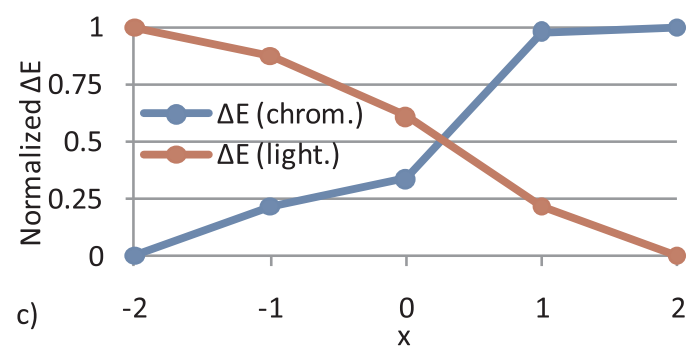

Figure 7: Analysis of selecting representative patch point. (a) Image of the target appearance. (b) Photos a compliant AEs with varying values for $x$ during AE patch creation. Selecting a representative patch point $r_{k}$ with a compliancy of $\bar{C}_{k}+x \sigma_{c_{k}}$ for $x<0$ yields very compliant colours at the expense of luminance. A $r_{k}$ near $\bar{C}_{k}+x \sigma_{c_{k}}$ for $x>0$ yields a brighter appearance but incorrect chromatic behavior (b).

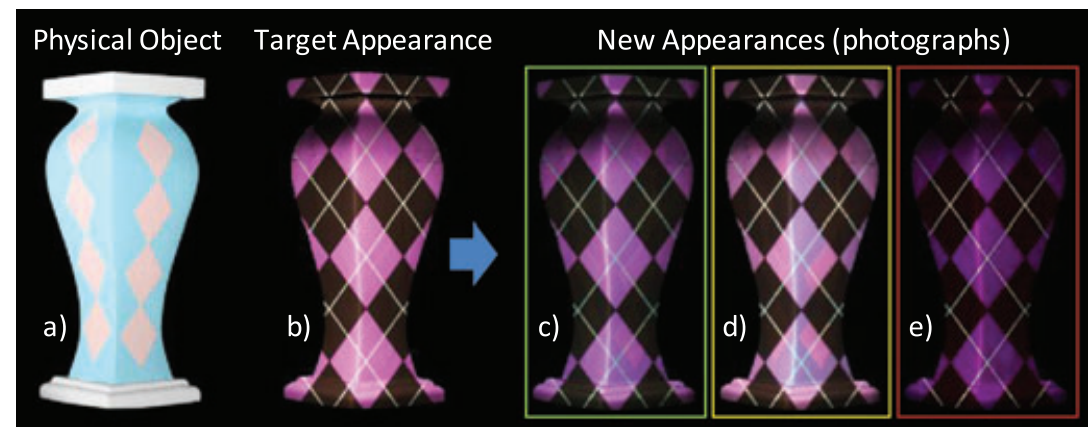

Figure 8: Complex albedo object. Photos of an AE on an object with a complex albedo. (a) Photo of the physical object. (b) Image of incompliant target appearance. (c) Our method produces a bright compliant AE. (d) AE naively using $A_{t}$ results in the surface albedo bleeding through. (e) Only reducing luminance to achieve compliancy results in a dim look.

a brighter solution, but because more patches will be seen as compliant prior to appearance optimization, a fewer number of incompliant patches steer the colour optimization, potentially resulting in unappealing colours and colour ratios. We choose a point with a compliancy value near $\bar{C}_{k}$ to obtain a reasonable balance- this corresponds to the intersection of the normalized luminance and chrominance error components in Figure 7(c).

In Figure 8, we physically change the surface albedo of the object used in Figure 5 to a more complex albedo pattern to demonstrate non-additive appearance changes (Figure 8a). The argyle pattern shown in Figure 8(b) is then imparted onto the object. Figures 8(c)-(e) contain photos of the resulting AEs. Naïvely using the incompliant target appearance results in the surface albedo's colour bleeding through and causing 'ghosting' patterns mostly on the bottom half of the object, achieving compliancy by only reducing the luminance of the appearance results in an overly dim look, and using our method results in a brighter, more perceptually similar compliant appearance. The small amount of 'ghosting', which remains is due to inaccuracies in the radiometric calibration rather than the compensation compliancy of the modified appearance.

Figure 9 shows the interdependency between patches on different objects. Compliancy is easy to achieve for the top half of both objects but not for the bottom halves. The compliant appearance obtained by reducing the luminance of the target image (Figure 9a) results in a dim look for the bottom of the left object (Figure 9c) when compared to our compliant solution (Figure $9 \mathrm{~b}$ ). A naïve AE using the original target appearance results in a colour shift to the bottom halves of both objects: the new appearance of the bottom of the left object is more beige and that of the bottom of the right object is more blue (Figure 9d). Our method balances the 


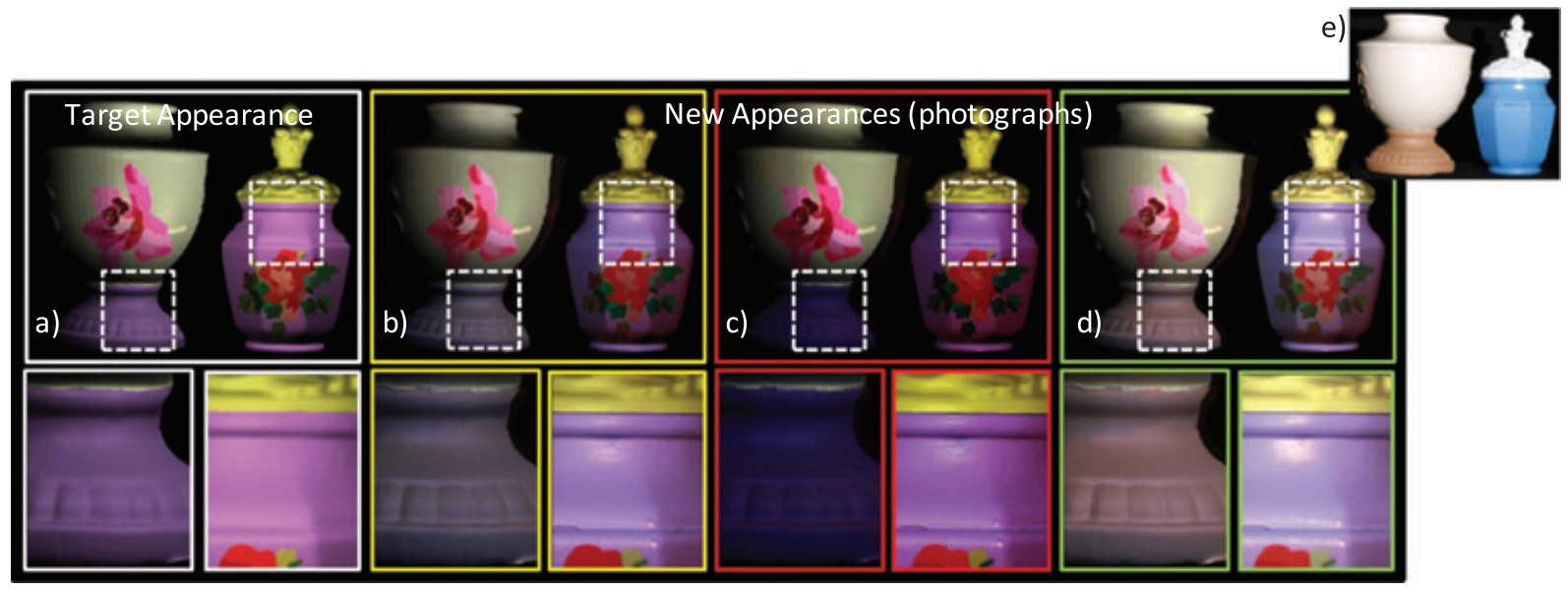

Figure 9: Two-object scene. (a) Incompliant target appearance (synthetic). (b) Photo of AE using our compliant appearance. (c) Photo of AE when reducing luminance until compliancy. (d) Photo of AE naïvely using target appearance. (e) Photo of the physical objects. This example shows the interdependency between patches of different objects. Although (c) maintains colours, it results in a dim look for some parts of the scene. In contrast, (d) is overall bright but has uncontrolled colour shifts. Our solution in (b) achieves a controlled balance.

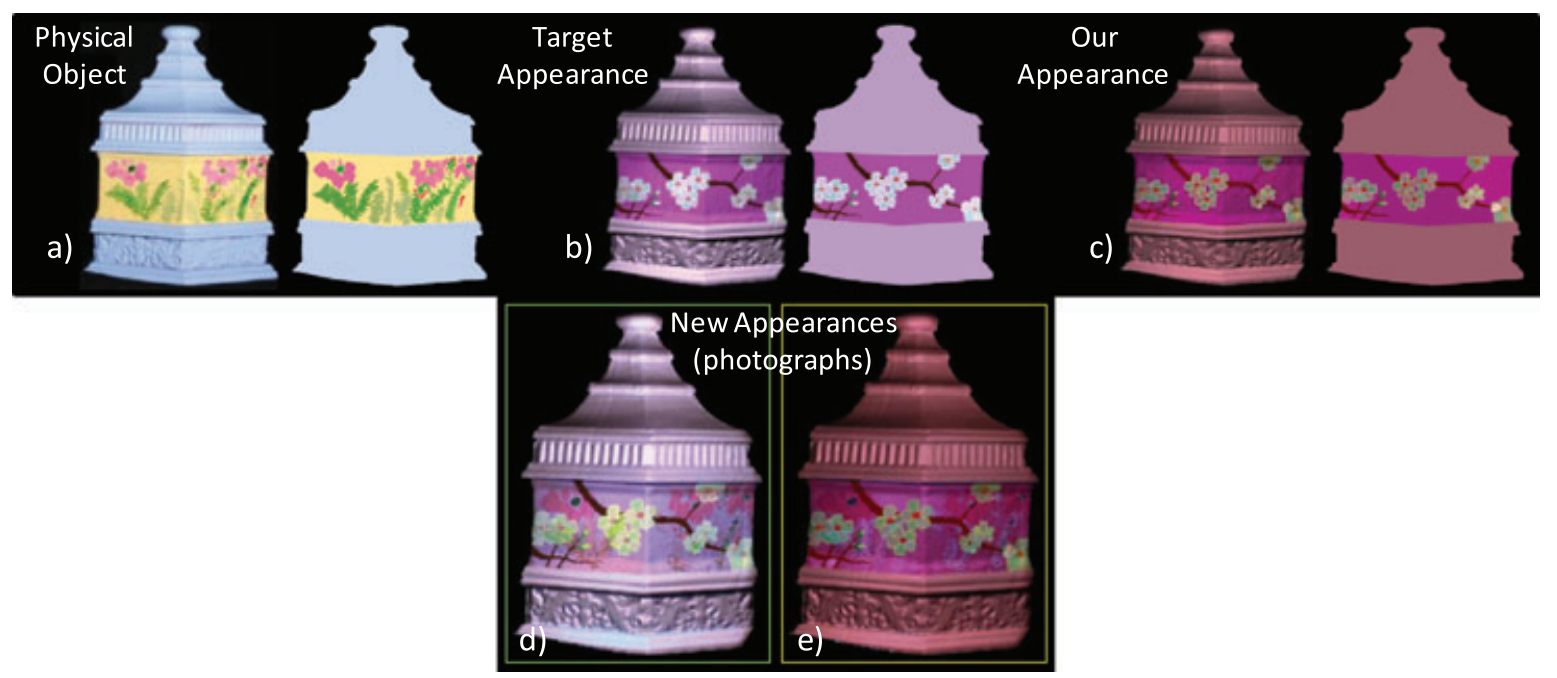

Figure 10: Extreme case. (a) Photo of physical object and computed surface albedo. (b) Incompliant target appearance and target albedo (both synthetic). (c) Our resulting appearance and albedo. d) Photo of AE naïvely using target appearance. (e) Photo of AE using our appearance. The strong colour change of the target appearance is severely limited by the underlying surface albedo. A naïve AE results in the surface colours bleeding through and in the relative colours of the target appearance not being achieved. Our appearance reduces colour bleeding and maintain the relative ratios (e.g. of hue, saturation, and contrast) of the appearance, however some 'ghosting' of the underlying surface albedo remains.

opposing chrominance and luminance requirements. Although a mild colour shift is present, patch colour ratios are mostly maintained.

Figure 10 demonstrates an $\mathrm{AE}$ of an object with a complex surface albedo needing an extreme amount of colour modification due to the large amount of light required to achieve the desired colours. The various albedos used are shown in Figures 10(a)-(c). Figure 10(d) shows a photo of a clearly incompliant AE produced by naïvely using the target appearance. Our resulting AE is shown in Figure 10(e). In this example, our resulting appearance significantly reduces the 'ghosting' of the underlying surface albedo shown in Figure 10(d) and better maintains the colour ratio of the 
top, middle and bottom bands of the object. However some 'ghosting' remains despite the appearance undergoing significant colour change. As discussed in Figure 7, selecting different $r_{k}$ 's with compliancy values less than $\bar{C}_{k}$ would produce a dimmer, more likely to be fully compliant appearance. Improved radiometric calibration which models colour mixing would help as well.

\section{Conclusions}

We have presented a novel method to achieve compliant AEs of physical objects using digital projectors. Our work addresses an area mostly overlooked by previous AE systems. Ignoring the compliancy of a target appearance might cause the produced appearance to significantly differ from what is expected, even with accurate geometric and radiometric calibration. Prior systems use white surfaces or assume a compliant target appearance. Our work considers both the chrominance and luminance components of the target appearance, encodes human perception cues into equations, and performs an appearance optimization to yield a perceptually similar, but compliant, appearance.

\section{Limitations and Future Work}

The main limitations of our method include the assumption that the scene is diffuse and the supposition that the object surfaces can be divided into patches of nearly constant colour. Although the first limitation can be addressed with improved acquisition and radiometric calibration, the latter poses more challenges. Using a different definition for patches (e.g. patches with chromatic patterns or a chromatic gradient) is an area of future work. Exploring the use of multiple surface points to represent a patch may also provide a more accurate representation of the object's surface, possibly improving the resulting compliant appearance. We would also like to support dynamically changing appearances. This would require accelerating the appearance modification compute time and incorporating temporal coherence to reduce unexpected colour shifts over time. We would like to explore $\mathrm{AE}$ in brightly lit rooms. $\mathrm{AE}$ is currently supported only in dark rooms. Non-trivial ambient light reduces the available range of colours, making dark colours impossible to achieve for a physically light surface patch. Finally, we would also like to conduct user studies to further evaluate our appearance modification method and to better understand the value of preserving colour constancy though changing absolute colour values.

\section{References}

[ALY08] Aliaga D., Law A., Yeung Y.: A virtual restoration stage for real-world objects. ACM Transactions on Graphics, 27, 5 (2008).

[BDEW01] Balasubramanian R., DeQueiroz R., Eschbach R., Wu W.: Gamut mapping to preserve spatial luminance variations. Journal of Imaging Science and Technology, 45, (2001), 436-482.

[BRF01] Bandyopadhyay D., Raskar R., Fuchs H.: Dynamic shader lamps: Painting on movable objects. In Proceedings of IEEE/ACM International Symposium on Augmented Reality (New York, NY, USA, 2001), IEEE Computer Society, 207-216.

[BI08] Bimber O. and IwaI D.: Superimposing dynamic range. ACM Transactions on Graphics, 27, 5 (2008).

[BIW08] Bimber O., Iwai D., Wetzstein G., Grundhoefer A.: The visual computing of projector-camera systems. Computer Graphics Forum, 27, 8 (2008), 22192245 .

[Bra99] Braun G.: A Paradigm for Color Gamut Mapping of Pictorial Images. Ph.D. thesis, Rochester Institute of Technology, 1999.

[BF99] Braun G., FAIRChILd M.D.: General-purpose gamut mapping algorithms: evaluation of contrast-preserving rescaling functions for color gamut mapping. Journal of Imaging Science and Technology (2000), 343-350.

[CM02] Comaniciu D., Meer P.: Mean shift: A robust approach toward feature space analysis. IEEE Transactions on Pattern Analysis and Machine Intelligence, 24, (2002), 603-619.

[GB08] GrundhöFer A., BIMBer O.: Real-time adaptive radiometric compensation. IEEE Transactions on Visualization and Computer Graphics, 14, 1 (2008), 97-108.

[GPNB04] Grossberg M. D., Peri H., Nayar S. K., Belhumeur P. N.: Making one object look like another: controlling appearance using a projector-camera system. In Proceedings of IEEE Conference on Computer Vision and Pattern Recognition, 1 (Washington, DC, USA, 2004), IEEE Computer Society, 452-459.

[HOK99] Hung-shing C., Omamiuda M., Kotera H.: Adaptive gamut mapping method based on image-todevice. Proceedings of IS\&Ts NIP 15, International Conference on Digital Printing Technologies (Orlando, FL, USA, 1999), Society for Imaging Science and Technology, 346-349.

[JF07] Johnson T., Fuchs H.: A unified multi-surface, multiresolution workspace with camera-based scanning and projector-based illumination. In Proceedings of Eurographics Symposium on Virtual Environments-Immersive Projection Technology Workshop (Weimar, Germany, 2007), Eurographics Association.

[KSES05] Kimmel R., Shaked D., Elad M., and Sobel I.: Space-dependent color gamut mapping: A variational

(c) 2011 The Authors Computer Graphics Forum (c) 2011 The Eurographics Association and Blackwell Publishing Ltd. 
approach. IEEE Transactions on Image Processing, 14, 6 (2005), 796-803.

[Lan77] LAND E. H.: The Retinex theory of color vision. Scientific American, 237, (1977), 108-128.

[LAM10] Law A., Aliaga D., Majumder A.: Projector placement planning for high quality visualizations on realworld colored objects, IEEE Transactions on Visualization and Computer Graphics, 16, 6 (2010), 1633-1641.

[LPA07] Lee B. S., Pizlo Z., Allebach J. P.: Characterization of red-green and blue-yellow opponent channels, Journal of Imaging Science and Technology, 51, 1 (2007), 23-33.

[MS05] Majumder A., Stevens R.: Perceptual photometric seamlessness in tiled projection-based displays. ACM Transactions on Graphics, 24, 1 (2005).

[NPGB03] Nayar S. K., Peri H., Grossberg M. D., Belhumeur P.N.: A projection system with radiometric compensation for screen imperfections. In Proceedings of ICCV Workshop on Projector-Camera Systems (Nice, France, 2003), IEEE Computer Society.

[OOD09] Okazaki T., Okatani T., Deguchi K.: Shape reconstruction by combination of structured-light projection and photometric stereo using a projector-camera system. In Proceedings of Pacific Rim Symposium on Advances in Image and Video Technology (Tokyo, Japan, 2009), Springer, 410-422.
[RWLB01] Raskar R., Welch G., Low K. L., BANDYOPADHYAY D.: Shader lamps: Animating real objects with image-based illumination. In Proceedings of Eurographics Workshop on Rendering Techniques (London, UK, 2001), Springer, 89-102.

[SLM10] Sajadi B., Lazarov M., Majumder A.: ADICT: Accurate direct and inverse color transformation. In Proceedings of European Conference of Computer Vision (Crete, Greece, 2010), Springer.

[SLMG09] Sajadi B., Lazarov M., Majumder A., Gopi M.: Color seamlessness in multi-projector displays using constrained gamut morphing, IEEE Transactions on Visualization and Computer Graphics, 15, 6 (2009).

[Sha03] Sharma G.: Digital Color Imaging Handbook (1.7.2 ed.). CRC Press, (2003).

[Wan95] Wandell B. A.: Foundations of Vision. Sinauer Associates, Inc., (1995).

[WB07] Wetzstein G. and Bimber O.: Radiometric compensation through inverse light transport. In Proceedings of Pacific Graphics, (Canmore, Alberta, Canada, 2003), IEEE Computer Society, 391-399.

[ZS07] ZolLiker P. and Simon K.: Retaining local image information in gamut mapping algorithms. IEEE Transaction on Image Processing, 16, 3 (2007), 664672 . 